\title{
Micro-engineered flexural post rings for effective blood sample fencing and high throughput measurement of clot retraction force
}

\author{
Supporting Document
}

Lanzhu Huang ${ }^{1,2, \#, ~ X i n y u ~ L i u ~}{ }^{1,2,3, \#, ~ Y u a n b i n ~ O u^{1,2}, \text { Haofan Huang }}{ }^{1,2}$, Xia Zhang ${ }^{4}$, Yize Wang $^{5}$, Yong Liang ${ }^{3}$, Xiaxia Yu ${ }^{1,2}$, Weidong Zheng ${ }^{4}$, Huisheng Zhang ${ }^{1,2}$, and Zida Li ${ }^{1,2, *}$

${ }^{1}$ Department of Biomedical Engineering, School of Medicine, Shenzhen University, Shenzhen 518060, China;

${ }^{2}$ Guangdong Key Laboratory for Biomedical Measurements and Ultrasound Imaging, Department of Biomedical Engineering, School of Medicine, Shenzhen University, Shenzhen 518060, China;

${ }^{3}$ Faculty of Information Technology, Collaborative Laboratory for Intelligent Science and Systems and State Key Laboratory of Quality Research in Chinese Medicines, Macau University of Science and Technology, Macao 999078, China;

${ }^{4}$ Department of Laboratory Medicine, Shenzhen University General Hospital, Shenzhen 518055, China;

${ }^{5}$ Department of Mechanical and Process Engineering, ETH Zurich, 8092 Zurich, Switzerland.

\#Contributed equally.

*Correspondence: zidali@szu.edu.cn 


\section{Supplementary Figures}

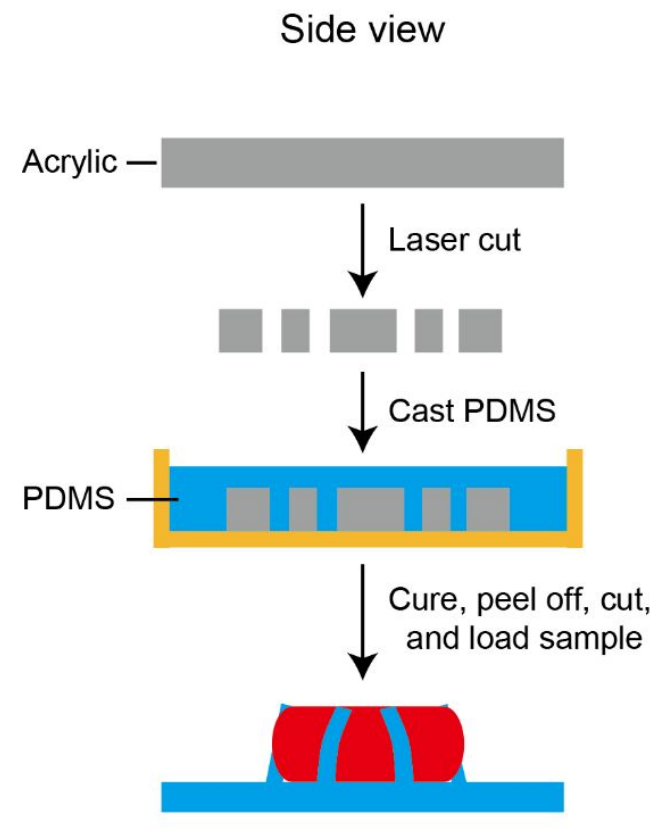

Perspective view

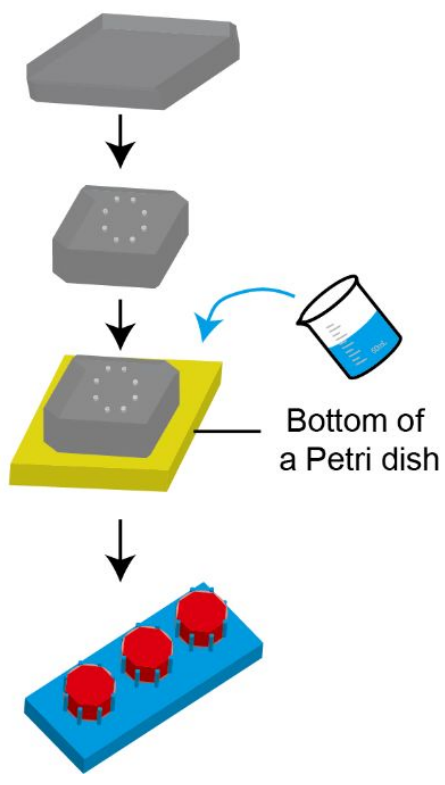

Figure S1. Fabrication process of the device. Acrylic sheet is cut by laser into desired shape and placed in a Petri dish to serve as a mold. PDMS replica molding is then used to fabricate the device. 


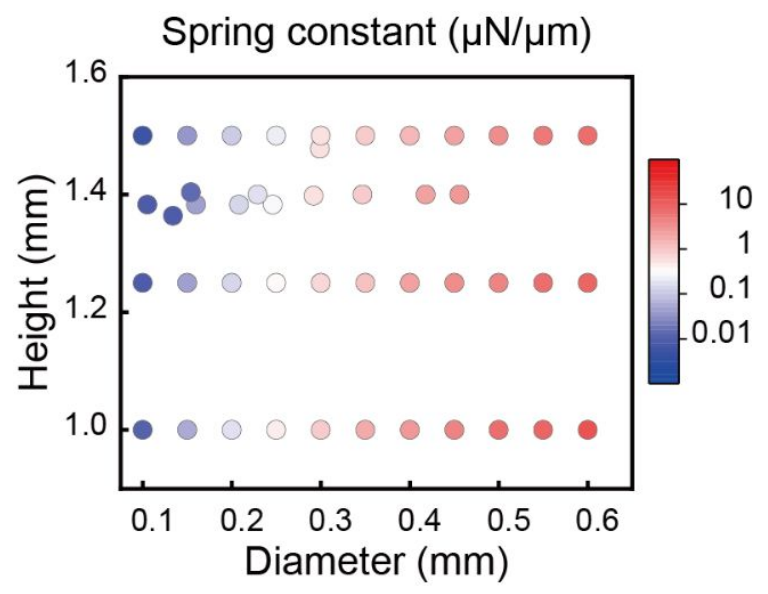

Figure S2. Spring constant of the post with different diameters and heights as calculated from the simulation. 


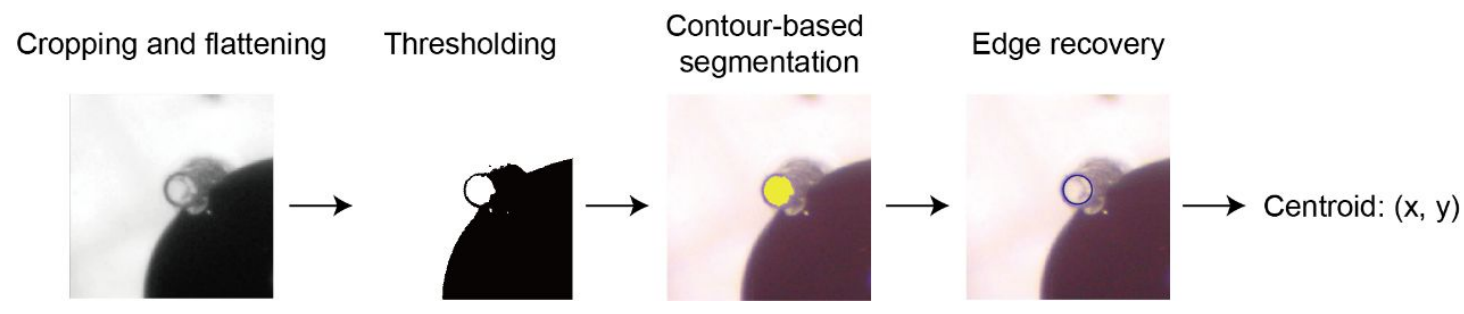

Figure S3. Algorithm of the image processing. Images are first cropped to segment individual post and flattened into greyscale images before they were thresholded to generate binary images. Contour analysis is then applied to locate the post tip, and the tip edge is recovered by fitting with a closing circle. The centroid of the circle is reported as the coordinate of the post tip. 

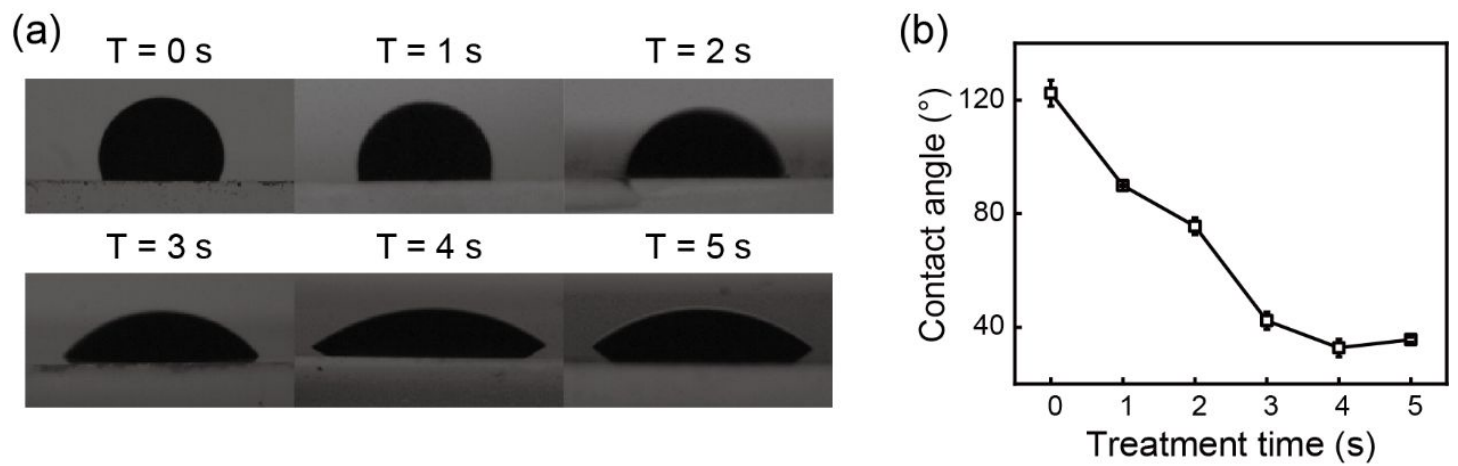

Figure S4. Measurement of the contact angles of surfaces treated with oxygen plasma of different dose. (a) Images of drops on surfaces treated with oxygen plasma for duration as indicated. (b) Contact angle as a function of oxygen-plasma treatment time. Data represent mean \pm S.D. with $\mathrm{n}=3$. 


\section{Supporting Table}

Table S1. Comparison of the measurements of baseline samples using flexural post rings and Hemodyne.

Method Flexural post ring Hemodyne

\section{Dimension of the gap \\ between opposing \\ anchors}

Sample preparation

Data sources

Peak force

Force per unit blood volume

$$
2.0 \mathrm{~mm}
$$

$4.5 \mu \mathrm{L}$ citrated whole blood, activated by calcium

biochemistry and

biophysics 38.1 (2003):

$55-78$

$$
43.6 \pm 2.7 \mu \mathrm{N}
$$

$46750 \pm 3278 \mu \mathrm{N}$

$$
9.7 \pm 0.6 \mu \mathrm{N} / \mu \mathrm{L}
$$

$66.8 \pm 4.7 \mu \mathrm{N} / \mu \mathrm{L}$

Table S2. Calibration of the electronic pipetting module.

\begin{tabular}{cc} 
Target volume $(\mu \mathrm{L})$ & $\mathrm{CV}(\%)$ \\
\hline 2.5 & 9.36 \\
4 & 3.5 \\
6 & 4.01 \\
8 & 2.24 \\
10 & 3.31
\end{tabular}




\section{Supporting Video}

Video S1. Demonstration of automated sample loading and image acquisition. 